\title{
Evaluating Medication Adherence: Which Measure Is Right for Your Program?
}

\begin{abstract}
OBJECTIVE: Medication-adherence programs are increasingly important to managed care organizations. This review discusses the strengths and limitations of medication-adherence measurement techniques, including biological markers, pill counts, electronic monitoring devices, patient surveys, and prescription claims data. Specific tools for measuring medication adherence using prescription claims data are defined and demonstrated. The process of selecting an appropriate tool is discussed.
\end{abstract}

DATA SOURCES: Published literature.
CONCLUSIONS: Prescription claims are an economical and timely data source for measuring medication adherence. While there are various measures that can be examined from claims data, prescription claims have limitations that must be considered in developing an evaluation plan.

KEYWORDS: Claims data, medication adherence, program evaluations

J Managed Care Pharm 2000: 499-504

\section{Authors}

KATHLEEN FAIRMAN MA, is Outcomes Research Manager and BRENDA MOTHERAL, Ph D, is Senior Director of Olitcomes Research, at Express Scripts, Inc, Tempe, AZ.

AUTHOR CORRESPONDENCE Kathleen Fairman, M.A., Outcomes Research Manager, Express Scripts, Inc, 1700 North Desert Drive, Tempe, AZ 85281, Tel 602-586-3167; E-mail: hfairman@express-scripts.com

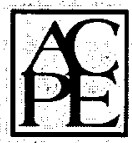

The Academy of Managed Care Pharmacy is approved by the American Council on Pharmaceutical Education as a provider. of continuing pharmaceutical education. Individuals may obtain up to 1 contact hour of credit or 0.10 Continuing Education Unit (CEU). The ACPE number is 233-000-00-006-HO1 Certificates will be mailed within eight weeks to participants who successfully complete the CE exam and achieve a score of $70 \%$ or more and submit the exam to AMCP prior to Decémber 31,2001 Learning objectives and test questions follow on page 505 Copynight 02000 Academy of Managed Care Pharmacy In Alt rights reserved.

\section{1}

by Kathleen Fairman and Brenda Motheral central element of many clinical programs within health plans. For example, some retrospective drug-utilization review (DUR) programs identify and intervene with patients who are considered to be noncompliant with their medication regimen. Increasingly, managed care organizations (MCOs) are implementing medication-adherence programs in which patients are encouraged, by telephone and/or mail, to continue to take medications that are to be used chronically. Therapeutic interchange programs, in which physicians and patients are asked to substitute a therapeutic alternative for their current medication, remain popular; the extent to which patients adhere to the substituted medication should be considered. At the core of many disease-management programs are educational and behavioral strategies to enhance medication adherence. Not only do MCOs want to examine the value of these programs, but they and the pharmaceutical companies have been challenged to demonstrate the extent to which such compliance programs are successful. Finally, medication-adherence measurement is becoming an increasingly important factor in debates about the appropriateness of various cost-containment strategies. $^{2-4}$

The purposes of this review are to highlight the advantages and disadvantages of various approaches to measuring medication adherence and to demonstrate some specific techniques for measuring medication adherence using prescription claims data. Pharmacists involved in the development and/or evaluation of medication-adherence activities will find this review relevant to their practice.

\section{Ways to Measure Adherence}

A commonly cited definition of compliance is "the extent to which a person's behavior coincides with medical or health advice." ${ }^{25}$ more recent years, the term has come into disfavor; the term adherence has been proposed as an altemative description.

While there are several methods for measuring medication adherence, none should be considered a gold standard. The selection of a measurement approach depends on the type of intervention being evaluated, the resources of the organization, and ethical and legal considerations related to patient intervention and confidentiality. As a general rule, it is important to remember that any approach that involves contacting patients (whether by mail, by telephone, or in person) adds complexity 
to a project. For example, reaching patients by telephone requires access to a telephone number database, as well as policies regarding patients whose phone numbers, although available in the health plan database, are unpublished (suggesting that the patient may prefer not to be contacted by telephone). Personnel are also required to research numbers that have changed since the database was assembled.

Enrolling patients into a formal research project requires legally and ethically acceptable informed-consent procedures, a timetable for contacts with patients (e.g., how many weeks after the start of treatment the initial contact will occur), and, usually, a budget to cover patients' costs. It is common for a project's true complexity to be undetected until the "nuts and bolts" of the project are worked through. Thus, no firm commitments to a project involving patient contact should be made until specific methods have been carefully considered.

Adherence measures fall into two general categories: direct and indirect measurements.6.7 Direct measurements, which include measures such as detection of the drug in biological fluid and direct observation of the patient taking medication, provide evidence that the patient took the medication. More commonly used are the indirect measurements, which include medication monitoring, self-report measures, and prescription claims data.

Medication monitoring can take the form of pill counts or the use of electronic monitoring devices. These devices record when the dosage form is accessed (e.g., a prescription bottle is opened or an inhaler is actuated). With self-report methods, patients may be asked to keep diaries of their medication use or to complete in-person, telephone, or mail surveys about their medication use.

As numerous authors have described, there are advantages and disadvantages to the various medication-adherence measures. ${ }^{6,7}$ Direct measures are considered by some to be more reliable and accurate than indirect measures, particularly patient report. ${ }^{8.9}$ However, they are labor-intensive and costly. Direct observation of medication-taking is usually not possible except in inpatient settings or unusual clinical trials. Measures from biological fluid provide evidence that the patient has taken the medication some time before the measurement takes place. However, they only measure adherence at one point in time, which is problematic for drugs with short half-lives, and they are subject to reliability problems because of variation in patients' metabolisms. ${ }^{8}$

As an indirect measure, pill counts can misrepresent adherence as well, particularly since they fail to measure whether medication was taken on schedule. ${ }^{8}$ They are used more often in formal research studies than in day-to-day health plan operations or ongoing program evaluations because they require the complexity of contacting and enrolling patients. Similarly, electronic monitoring devices are less obtrusive and generally more accurate than pill counts but are not trivial in expense. ${ }^{6}$
Self-report measures, if they are used to identify reasons for nonadherence, present the advantage of obtaining information from the patient's perspective, a.viewpoint that is increasingly being recognized as important. ${ }^{7,10-12}$ However, both diaries and, to a lesser extent, patient surveys require some effort to enroll patients. Additionally, both measures are considered unreliable by some because of patient recall and bias issues, although selfreported compliance has been linked to long-term health outcomes for patients with hypertension. ${ }^{78.13}$ Patient diaries may be more of an intervention than a measurement tool (because the patient is completing the diaries and taking the medication concomitantly), and surveys conducted while treatment is ongoing can also create a "Hawthorne effect" (the tendency of patients to increase compliance because they know they are being observed).

No gold-standard questionnaire for measuring patient adherence currently exists, although there are some validated questionnaires available. ${ }^{13}$ If an organization decides to use a self-report measure, careful questionnaire timing (post-treatment is better than during treatment) and item construction will substantially reduce bias. A particular focus of survey-item development should be to avoid the implication that noncompliant patients are in some way derelict. Another important focus is to replace subjective measures with objective measures as much as possible. For example, a question like "Did you take all medication as prescribed by your doctor?" is best replaced by "Some people take all the medication in the bottle during treatment, while others do not. What about you? Is your bottle of [medication name] empty or does it still have one or more pills in it?" The first statement lets the respondent know that all possible answers are acceptable. The second gives the respondent a specific objective standard, rather than the vague standard expressed in the original wording. For further information on the use of patient surveys in MCOs, see a review article that was recently published in this journal. ${ }^{14}$

Use of prescription claims data presents its own set of strengths and limitations. One of the biggest advantages of using prescription claims data is that the "Hawthorne effect" is avoided. For many MCOs, claims data are relatively accessible and inexpensive compared to other medication-adherence measurements. The reliability and validity of prescription claims data has been examined in numerous studies in the United States and Canada. These studies have found pharmacy claims-based measures of adherence to be a reliable source of drug exposure as estimated by home inventories and physician office records, to correlate with drug effects (e.g., blood pressure, phenytoin drug levels), and to be more complete than prescription use documented in medical records. ${ }^{15-20}$ However, claims data have the major disadvantage that they can be used only to study chronic, not acute, treatments. This limitation occurs because all claims-based measures of adherence rely on identifying prescription refills. For example, a researcher can 
Evaluating Medication Adherence: Which Measure Is Right for Your Program?

TABLE 1

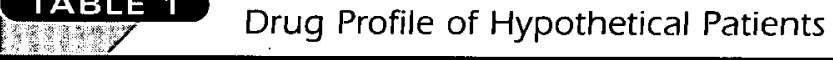

Patient A

\begin{tabular}{l|c|c|c|c}
\hline Drug & Quantity & Days & Date & Days Elapsed \\
\hline Enalapril 10 mg & 90 & 90 & $1 / 10 / 99$ & 0 \\
Enalapril 10 mg & 90 & 90 & $4 / 12 / 99$ & 92 \\
Enalapril 10 mg & 90 & 90 & $7 / 14 / 99$ & 185 \\
Enalapril 10 mg & 30 & 30 & $10 / 29 / 99$ & 292 \\
\hline
\end{tabular}

Patient B

\begin{tabular}{l|c|c|c|c}
\hline Drug & Quantity & Days & Date & Days Elapsed \\
\hline Enalapril 10 mg & 60 & 30 & $1 / 10 / 99$ & 0 \\
Enalapril 10 mg & 90 & 45 & $2 / 22 / 99$ & 43 \\
Enalapril 10 mg & 60 & 30 & $4 / 15 / 99$ & 95 \\
Enalapril 10 mg & 60 & 30 & $5 / 17 / 99$ & 127 \\
Enalapril 10 mg & 60 & 30 & $7 / 2 / 99$ & 173 \\
Enalapril 10 mg & 60 & 30 & $8 / 19 / 99$ & 221 \\
\hline
\end{tabular}

identify discontinuation of a chronic medication if a patient initially fills a prescription for a 15-day supply of chronic medication and then never refills the prescription. However, if the medication was intended to be taken for only 15 days (e.g., an antibiotic), no refill is expected for most patients. Thus, there is no way to tell from the claims data whether the patient took the medication for 7 days, 10 days, or all 15 .

\section{Dataset Preparation}

Prior to beginning an analysis of medication adherence using claims data, it is important to check for outliers and clinically impossible values. For example, a claim for a quantity of 30 Prozac $40 \mathrm{mg}$ tablets with a days supply of three would suggest a daily dosage of $400 \mathrm{mgs} /$ day. A likely explanation is that the pharmacist made a keying error and the actual days supply is 30. To check for problems of this type, use the formula $\mathrm{DD}=(\mathrm{QxS}) / \mathrm{DS}$, where $\mathrm{DD}=$ daily dose, $\mathrm{Q}=$ quantity dispensed, $\mathrm{S}=$ strength, and DS=days supply.

The vast majority of values will fall into standard dosing ranges. For those that do not, there are two possible approaches. One is to attempt correction of the claims by making assumptions about what the pharmacist intended to do (e.g., the days supply figure of 3 is really 30 ). This approach is both time-consuming and risky, since one cannot always accurately guess someone else's intentions. Another approach that is recommended, if sample size is sufficient, is to remove from the study sample any person who has at least one claim with a clearly incorrect value. An incorrect approach is to remove only the incorrect $\operatorname{claim}(\mathrm{s})$; that approach would remove part of the patient's history and therefore overestimate nonadherence.

Another important aspect of dataset preparation is control for eligibility. For example, if a patient has claims from January through March of 1999, and is followed through December of 1999 with no additional claims, it is important to know whether the patient was eligible during that time. If ineligible, the patient might in fact have continued taking the medication under a different payor's prescription card. For this reason, only patients who are continuously eligible throughout the study period should be used in studying medication adherence.

\section{Explanation of Measures}

Figure 1, page 503, shows the types of information that can be found in claims data and the supporting member, physician, and pharmacy claims. When using prescription claims data, there are a number of different medication adherence measures that can be examined, including, but not limited to, length of therapy, persistence, days of coverage, gaps, and medication possession ratios. In describing each of these measures, the prescription claims history for Patient $A$ and Patient $B$, as shown in Table 1 , left, will be used. Table 1 shows information that can be found in prescription claims data, including the drug name and strength, quantity of medication, days supply as keyed in by the pharmacist, and the date the prescription was filled. The last column shows the number of days elapsed since the first prescription during 1999 for each subsequent claim. Hypothetical Patient A had a total of four claims for enalapril during 1999, each for a 90-day supply until the final claim, which was for a 30-day supply. It appears from the quantity and days-supply figures that Patient A was taking enalapril once a day. Hypothetical Patient B had six claims for enalapril during 1999 and appeared to be taking the medication twice a day.

\section{Length of Therapy}

Length of therapy, which is a continuous measure, is the number of days elapsed between the date of the first claim for the drug of interest and the date when the days supply of the last claim is depleted. For Patient $A$, length of therapy equals the estimated final depletion date (date of the last claim for enalapril plus the days supply of the last claim) minus the date of the first claim $(292+30)$ or 322 . For Patient B, length of therapy equals $251(221+30)$. For patients who stop taking their medication before the supply of their last claim runs out, this measure will overestimate true length of therapy. For example, although Patient B received a 30-day supply of medication on August 19, he or she may have stopped taking the medication after 10 days and discarded the remaining medication, which would make the true length of therapy 231 days.

\section{Persistence}

Persistence, which is a dichotomous yes/no measure that is based on the length of therapy, tells whether a patient's length 
of therapy meets or exceeds a certain threshold. For example, at 90 days, Patient $A$ and Patient $B$ are persistent because their lengths of therapy are greater than 90 days. At 270 days, Patient $B$ is not persistent while Patient $A$ is. Persistence is most effectively used when evaluating treatment length against a clearly defined and unequivocal standard. For example, clinicians would uniformly agree, that antidepressant treatment of less than one month for patients diagnosed with depression is insufficient. Thus, a finding that $25 \%$ of initially treated patients fail to fill a second 30-day prescription would be important.

\section{Days of Coverage}

The days of coverage is simply the total days supply of medication that the patient had on hand during the year, as evidenced from the pharmacy claims. In 1999, Patient A had a total of 300 days supply of medication on hand $(90+90+90+30)$ while Patient $B$ had a total of 195 days supply on hand $([30 \times 5]+45)$. A limitation of this measure when individual claims are not reported is that in cases such as Patient B's, one does not know whether the 195 days supply was spread out over the entire year or whether the patient discontinued the medication altogether earlier in the year. This distinction may be important in certain situations.

\section{Gaps}

A gap, which is calculated for refill prescriptions, is the number of days between the assumed depletion date of one claim (the claim's fill date plus days supply) and the fill date of the next refill. The gap between Patient A's first and second claim was 2 days (first prescription was depleted in 90 days and the next fill occurred on the 92 nd day). The gap between the second and third claim was 3 days $(185-[92+90])$, and the gap between the third and fourth claim was 17 days $(292-[185+90])$. Thus, the mean gap was 7.33 days $([2+3+17] / 3)$, and the median gap was 3 days. For Patient $B$, the gaps were $13,7,2,16$, and 18 days, resulting in a mean gap of 11.2 days and a median gap of 13 days

In calculating gaps, one assumes that the medication is taken as initially prescribed until consumed. This assumption might be incorrect, because patients who reduce their dosage extend their period of medication-taking beyond the initial estimated depletion date. For example, if a patient is initially prescribed $100 \mathrm{mgs} / \mathrm{day}$ for 30 days, but reduces the daily dosage to $50 \mathrm{mgs}$ halfway through the treatment period, the prescription will last for 45 days ( 15 days at $100 \mathrm{mgs}+30$ days at $50 \mathrm{mgs}=$ the 3,000 $\mathrm{mgs}$ that were initially dispensed). In this situation, the patient is taking medication throughout the time period without any actual gaps in medication use. Obviously, such situations do not necessarily represent nonadherence since physicians sometimes prescribe dosage reductions after the prescription is dispensed. Fairman found that early refill of antidepressants were associated with dosage increases, while late refills were associated with dosage reductions. Christensen et al. reported a similar finding. ${ }^{22}$

\begin{tabular}{|c|c|c|}
\hline & Patient A & Patient B \\
\hline Length of therapy & 322 days & 251 days \\
\hline Persistent at nine months & yes & no \\
\hline Days of coverage & 300 & 195 \\
\hline Median gap & 3 & 13 \\
\hline MPR with therapy denominator & 0.92 & 0.75 \\
\hline MPR with calendar denominator & 0.82 & 0.53 \\
\hline
\end{tabular}

\section{Medication Possession Ratio}

The medication possession ratio (MPR) is calculated as the sum of the days supply for all claims during a defined period of time divided by the number of days elapsed during the period. ${ }^{23-25}$ The time period used in the denominator for this calculation is usually either the length of therapy or a study period of eligibility that is the same for every person in the sample. ${ }^{23-25}$ The conclusions reached in an MPR analysis depend heavily upon the choice of denominator. For example, assume that Patient A and Patient B are both eligible throughout calendar year 1999. In a calculation based on length of therapy, the denominator can be defined as the number of days elapsed between the fill date of the first and last prescription claim during the period, and the numerator can be defined as the days supply for all claims prior to the final fill date. Using this definition, Patient A's MPR $=270 / 292=0.92$, and Patient B's MPR $=165 / 221=0.75$.

An alternate method is to define the denominator as the total days in the study period. ${ }^{24}$ During calendar year 1999 , Patients $A$ and B receive a total of 300 and 195 days of medication, respectively. When the entire one-year study period is used as the denominator, Patient A's MPR is $0.82(300 / 365)$ and Patient $B$ 's is 0.53 (195/365). Thus, when the denominator is the entire calendar year, both patients, particularly Patient B, appear to be less compliant than when duration of therapy is used as the denominator. The reason is that when duration of therapy is used as the denominator, the MPR is affected solely by gaps from fill to refill. When the entire calendar period is used, the MPR is affected both by gaps and by terminations of treatment. Thus, the MPR is not useful in many situations in which one wants to get a clear picture of the nature of nonadherence, including both premature terminations and gaps.

Another major advantage of a gap analysis over an MPR analysis is that MPRs provide only a global, and sometimes misleading, picture of adherence. For example, assume that over a 12-month treatment period, a patient refills every prescription four days late. The days of medication obtained is $365-48=317$. The MPR is 86.8 (317/365), which seems low. However, if the medication half-life is such that a 4-day gap in treatment is clinically inconsequential, the MPR of 86.8 is not a concern. To obtain the relevant information from a gap analysis requires the calculation of 


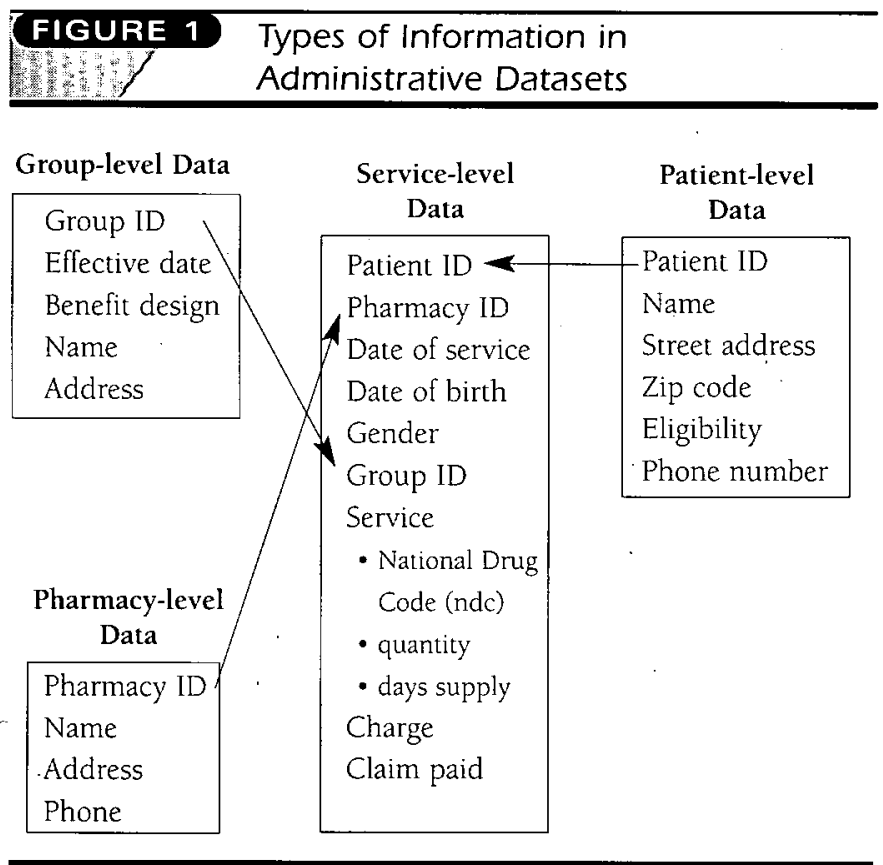

not only a mean (average) but also measures of distribution, for example, percentiles (usually 5th, 10th, 25th, 50th (median), 75th, 90th, and 95th), minimum, and maximum.

Table 2, page 502, summarizes each of the medication adherence measures that were discussed. Patient A performs better on all adherence measures. However, this is not always the case, as a patient may have a high length of therapy but have a relatively low MPR during the time period. Different measures yield different answers.

\section{Selection of Medication-adherence Measures from Pharmacy Claims}

Which measures should be examined in a particular situation? In any situation in which one is trying to determine the nature and extent of nonadherence, all of them should be calculated. One of the most important of these situations is the design phase of a medication-adherence program; one cannot effectively target a problem until one knows what the problem is. Then, which measures become the focus of policy change or corrective action depend both on clinical features of the medication class being studied and on the objective of one's measurement. For example, assume that you are trying to identify patients to enroll in a medication adherence program for a drug that requires continuous blood levels to be effective. A finding that $95 \%$ of patients continue treatment for an appropriate length of time, but frequently fill prescriptions late, suggests that a refill reminder program is an appropriate corrective activity. In contrast, a finding that $50 \%$ of patients discontinue medication prematurely suggests the need for education early in treatment. A similar approach should be used in program eval- uations; the selection of measure(s) again depends upon the goal of the program. Many medication-adherence programs are aimed at encouraging patients not to discontinue their chronic medications altogether, so length of therapy or even simpler persistence measures should be sufficient evidence of program success for those cases. For an asthma program whose goal is for patients to use their inhaled steroid on a regular basis rather than just when they have exacerbations, days of coverage and MPRs will be a more appropriate choice to demonstrate program success. In some instances, such as antiepileptic medications, a program might target drug holidays, which are patientinitiated holidays from drug therapy-defined as three or more days. ${ }^{26}$ Gap analysis could be used to determine whether the program successfully reduced the number of drug holidays.

\section{Limitations of Pharmacy Claims Data for Measuring Adherence}

Pharmacy claims data are not a good data source for measuring adherence with all drug therapies. As mentioned previously, it is not possible to measure adherence from the existence of one claim for an acute medication. Additionally, when the days-supply figure is unreliable, prescription claims data will not be a good choice if the measures are not interpreted appropriately. Days supply is more likely to be unreliable with injectable, transdermal, and inhaler dosage forms because of the inability to quantify the amount of medication contained in a single dose ${ }^{27}$ An option is to conduct sensitivity analysis of the days supply based on recommended guidelines for appropriate use of the medications. If the amount of medication to be used is questionable, as may be the case with insulins, or the directions for use allow for patient discretion (e.g., "prn"), the days supply figure is likely to be unreliable. For medications that require frequent dosage changes, such as oral anticoagulants, the use of claims data should be avoided. ${ }^{19}$ Finally, in cases where medication stockpiling and/or sharing with family and friends is particularly likely to occur (e.g., nonsedating antihistamines), pharmacy claims data may not provide a particularly meaningful measure of medication adherence.

In addition to considering, whether pharmacy claims data are an appropriate source for the medication one is considering, there are other caveats to be aware of in calculating and interpreting these adherence measures from pharmacy claims data. When calculating adherence measures, such as gaps and medication possession ratios, the time period of observation is an important consideration. Early in therapy, when dosages are more likely to be adjusted after a prescription has been dispensed, it may be difficult to measure gaps accurately. Christensen et al. found that the extent of overcompliance (a situation in which the number of days dispensed exceeds the length of the study period) diminished with longer observation periods and recommended a minimum of 60 , and preferably 90 days for observation. ${ }^{22}$ 
Not all medication adherence measures can be examined with claims data. As discussed earlier, claims data do not indicate the timing of doses; in cases in which timing is of critical importance, prescription claims data will not be an appropriate choice. ${ }^{28}$ Furthermore, for drugs with long half-lives, as mentioned previously, gaps in therapy may not lead to therapeutic failure. Clearly, pharmacy claims measures do not address whether or not the patient filled the first prescription that was written for a particular medication. Finally, in cases in which the patient is more likely to purchase medication outside the pharmacy network, the use of pharmacy claims data becomes problematic. For example, it is not possible to study adherence with diphenhydramine treatment, because this product is readily available over the counter.

\section{Conclusions}

Pharmacists in managed care settings are increasingly involved in efforts to identify and improve patient medication adherence. Doing so requires an understanding of the various types of medication adherence measures and the sources of measurement. Although not appropriate for every situation, pharmacy claims data represent an economical and valid source of measurement when used appropriately. Pharmacists should understand the strengths and limitations of pharmacy claims to make effective use of this data source.

\section{References}

1. Reissman D. Back to compliance. Drug Benefit Trends 1998; 10 (10), 18.

2. Johnson RE et al. The impact of increasing patient prescription drug cost sharing on therapeutic classes of drugs received and on the health status of elderly HMO members. Health Services Research 1997; 32: 103-22.

3. Motheral BR, Henderson R. The effect of a closed formulary on prescription drug use and cost. Inquiry 1999-2000; 36: 481-91.

4. Reeder CE et al. Economic impact of cost-containment strategies in third party programmes in the US (Part 1). Pharmacoeconomics 1993; 4: 92-103.

5. Haynes RB. Introduction in compliance in health care. eds Haynes RB, Taylor DW, Sackett DL. Baltimore: The Johns Hopkins University Press, 1979; $1-7$.

6. Farmer $\mathrm{KC}$. Methods for measuring and monitoring medication regimen adherence in clinical trials and clinical practice. Clinical Therapeutics 1999; 21; 6: 1071-90.

7. Morris LS, Schulz RM. Patient compliance: an overview. J Clin Pharmacy and Therapeutics 1992; 17: 283-95.

8. Cramer JA et al. How often is medication taken as prescribed?: A novel assessment technique. JAMA 1989; 261: 3273-77

9. Boudes P. Drug compliance in therapeutic trials: A review. Controlled Clinical Trials 1998; 19: 257-68.

10. Arluke A. Judging drugs: Patients' concepts of therapeutic efficacy in the treatment of arthritis. Human Organization 1980; 39: 84-88.

11. Conrad P. The meaning of medications: Another look at compliance. Social Science and Medicine 1985; 20: 29-37.

12. Rich $\mathrm{M}$ et al. Asthma in life context: Video intervention/prevention assessment. Pediatrics 2000; 105: 469-77.

13. Morisky DE, Green LW, Levine DM. Concurrent and predictive validity of a self-reported measure of medication adherence. Medical Care 1986; 24: 67-74.

14. Fairman KA. Going to the source: A guide to using surveys in health care research. J Managed Care Pharm 1999; 5(2): 150-59.

15. Lau HS et al. Validation of pharmacy records in drug exposure assessment. J Clinical Epidemiology 1997; 50(5): 619-25.

16. Johnson RE, Volmer WM. Comparing sources of drug data about the elderly. J Amer Geriatric Society 1991; 39: 1079-84.

17. Tamblyn $\mathrm{R}$ et al. The use of prescription claims databases in pharmacoepidemiological research: The accuracy and comprehensiveness of the prescription claims database in Quebec. J Clinical Epidemiology 1995; 48(8): 999-1009.

18. Steiner JF et al. A general method of compliance assessment using centralized pharmacy records. Medical Care 1988; 26: 814-23.

19. Steiner JF, Prochazka AV. The assessment of refill compliance using pharmacy records: Methods, validity, and applications. J Clinical Epidemiology 1997; 50(1): 105-16.

20. Kirking DM, Ammann MA, Harrington C. Comparison of medical records and prescription claims files in documenting prescription medication therapy. J Pharmacoepidemiology 1996; 5(1): 3-15.

21. Fairman KA. "The doctor told me to cut the pills in half": Practical considerations in using claims databases for outcomes research. Drug Benefit Trends 1997; 9(10): 30-35, 39.

22. Christensen DB et al. Assessing compliance to antihypertensive medications using computer-based pharmacy records. Medical Care 1997; 35(11): $1164-70$.

23. Sclar DA et al. Antidepressant pharmacotherapy: Economic outcomes in a health maintenance organization. Clinical Therapeutics 1994; 16: 715-30.

24. Okano GJ et al. Patterns of antihypertensive use among patients in the US Department of Defense database initially prescribed an angiotensin-converting enzyme inhibitor or calcium channel blocker. Clinical Therapeutics 1997; 19: 1433-45.

25. Blandford $\mathrm{L}$ et al. Analyzing variations in medication compliance related to individual drug, drug class, and prescribing physician. J Managed Care Pharm 1999; 5: 47-51.

26. Urquhart J, Chevalley C. Impact of unrecognized dosing errors on the cost and effectiveness of pharmaceuticals. Drug Information J 1988; 22: 363-78.

27. Farris KB, Kaplan B, Kirking D. Examination of days supply in computerized prescription claims. J Pharmacoepidemiology 1994; 3(1): 63-76.

28. Choo PW et al. Validation of patient reports, automated pharmacy records, and pill counts with electronic monitoring of adherence to antihypertensive therapy. Medical Care 1999; 37(9): 846-57. 


\section{E E X A M}

Evaluating Medication Adherence: Which Measure Is Right for Your Program?

Upon completion of this article, the successful participant should be able to:

1. Identify the various reasons for which health care organizations are using medication-adherence measures.

2. Discuss the advantages and disadvantages of direct and indirect measures of medication adherence.

3. Describe specific measures of medication adherence calculated from prescription claims data.

4. Explain appropriate uses of prescription claims data for medicationadherence measurement.

\section{SELF-ASSESSMENT QUESTIONS}

1. Activities for which measuring medication adherence is important include:
a. retrospective drug utilization review.
b. pharmacy benefit design changes.
c. disease management.
$\mathrm{d}$. all of the above.

2. An example of a direct medicationadherence measure is:
a. detection of drug in biological fluid.
b. pill counts.
c. electronic monitoring devices.
d. patient surveys.

3. An example of an indirect medication-adherence measure is:
a. pill counts.
b. electronic monitoring devices.
c. prescription claims data.
d. all of the above.

4. An advantage of direct measures is that they:

a. measure adherence at a point in time.

b. provide evidence that the patient actually took the medication.

c. are subject to reliability problems because of variations in patients metabolisms.

d. are very inexpensive.

5. The gold standard for measuring medication adherence is:
a. electronic monitoring devices
b. patient surveys.
c. prescription claims data.
d. nonexistent.

6. For which of the following data sources is the "Hawthorne effect" NOT a potential limitation?
a. patient surveys
b. patient diaries
c. prescription claims data
$\mathrm{d}$. none of the above

7. Which one of the following statements about measuring length of therapy using claims data is NOT true?

a. It is a dichotomous (yes/no) measure.

b. It will overestimate use for patients who stop taking their medication before the supply of their last claim runs out.

c. It is the number of days between the date of the first claim and the depletion date of the last claim for the drug of interest.

d. It can be used to determine persistency at various points in time.

8. Which one of the following statements about the calculation of medication gaps is true?

a. It equals the total days supply of medication over the time period.

b. It assumes that the medication is taken as prescribed until consumed.

c. It is not affected by medication dosage changes.

$\mathrm{d}$. It is a measure of medication continuation.

9. An inappropriate data source for measuring adherence with acute medications is:
a. patient surveys.
b. pill counts.
c. prescription claims data.
d. electronic monitoring devices.

10. Medications for which the days supply figure is likely to be unreliable include:
a. injectables.
b. inhalers.
c. "prn" medications.
d. all of the above. 


\section{DEMOGRAPHIC INFORMATION (not for scoring)}

11. In what type of setting do you work? (Leave blank if none of the responses below applies.)
a. $\mathrm{HMO}$
b. $\mathrm{PPO}$
c. Indemnity insurance
d. Pharmacy benefits management
e. Other

12. Did this program achieve its educational objectives?
a. Yes
b. No

13. How many minutes did it take you to complete this program, including the quiz? (Fill in on answer sheet.)
14. Did this program provide insights relevant or practical for you or your work?
a. Yes
b. No

15. Please rate the quality of this $C E$ article.
a. Excellent
b. Good
c. Fair
d. Poor

\section{INSTRUCTIONS}

$\mathrm{PE}$ This test affords 1 hour $(0.10 \mathrm{CEU})$ of continuing pharmaceutical education in all states that recognize the American Council on Pharmaceutical Education. To receive credit, you must score at least $70 \%$ of your test answers correctly. To record an answer, darken the appropriate block below. Mail your completed answer sheet to: Academy of Managed Care Pharmacy, $100 \mathrm{~N}$. Pitt Street, Suite 400 , Alexandria, VA 22314. If you score $70 \%$ or more, a certificate of achievement will be mailed to you within eight weeks. If you fail to achieve $70 \%$ on your first try, you will be allowed only one retake. The ACPE Provider Number for this lesson is 233-000-00-006-H01. This offer of continuing education credit expires December 31, 2001.
A $\quad$ B $\quad$ C $\quad$ D
1. $コ$ व
A $\quad$ B $\quad$ C $\quad$ D
2. $\square \quad \square \quad \square \quad \square$
3. $\square \quad \square \quad \square \quad \square$
4. $\square \quad \square \quad \square \quad \square$
5.

$\begin{array}{llll}\square & \square & \square\end{array}$
6. $\square \square \square \square$
7. $\square \quad \square \quad \square \quad \square$
8. $\square \quad \square \quad \square \quad \square$
9. $\square \quad \square \quad \square \quad \square$
10. $\square \quad \square \quad \square \quad \square$

11. $\square \mathrm{A} \quad \square \mathrm{B} . \quad \square \mathrm{C} \quad \square \mathrm{D} \quad \square \mathrm{E}$

12. $\square$ Yes $\square$ No

13. Minutes

14. $\square$ Yes $\square$ No

15. $\square \mathrm{A} \quad \square \mathrm{B} \quad \square \mathrm{C} \square \mathrm{D}$

Participant Identification: Please type or print.

Date:

Social Security \#:

For Identification Purposes Only

Work Phone \#:

Name:

LAST FIRST MIDDLE

Company:

Address:

STREET (with Apt. No.) or P.O. Box

STATE .

ZIP

State \& Lic. No.:

STATE LICENSE NO.

Member Type: $\square$ Active

$\square$ Supporting Associate

$\square$ Student

Nonmember

Signature:

I verify by my signature above that I have completed this examination independently. 